\title{
Evolution of spin direction of accreting magnetic protostars and spin-orbit misalignment in exoplanetary systems
}

\author{
Dong Lai ${ }^{1,2}$, Francois Foucart ${ }^{1}$ and Douglas N. C. Lin ${ }^{3,4}$ \\ ${ }^{1}$ Center for Space Research, Department of Astronomy, Cornell University, Ithaca, NY 14853 \\ email: dong@astro.cornell.edu \\ ${ }^{2}$ Kavli Institute for Theoretical Physics, University of California, Santa Barbara, CA 93106 \\ ${ }^{3}$ Department of Astronomy and Astrophysics, University of California, Santa Cruz, CA 95064 \\ ${ }^{4}$ Kavli Institute for Astronomy and Astrophysics, Peking University, Beijing, China
}

\begin{abstract}
Recent observations have shown that in many exoplanetary systems the spin axis of the parent star is misaligned with the planet's orbital axis. These have been used to argue against the scenario that short-period planets migrated to their present-day locations due to tidal interactions with their natal discs. However, this interpretation is based on the assumption that the spins of young stars are parallel to the rotation axes of protostellar discs around them. We show that the interaction between a magnetic star and its circumstellar disc can (although not always) have the effect of pushing the stellar spin axis away from the disc angular momentum axis toward the perpendicular state and even the retrograde state. Planets formed in the disc may therefore have their orbital axes misaligned with the stellar spin axis, even before any additional planet-planet scatterings or Kozai interactions take place. In general, magnetospheredisc interactions lead to a broad distribution of the spin-orbit angles, with some systems aligned and other systems misaligned.
\end{abstract}

Keywords. accretion, accretion disks, planetary systems: protoplanetary disks, stars: magnetic fields

\section{Introduction}

As discussed by others in this conference (J. Winn, A. Triaud), the Rossiter-McLaughlin (RM) effect, an apparent radial velocity anomaly caused by the partial eclipse of a rotating parent star by its transiting planet, can be used to measure the sky-projected stellar obliquity, the angle between the stellar spin axis and the planetary orbital axis. Among the dozens of systems with RM measurements, about $60 \%$ have an orbital axis aligned (in sky projection) with the stellar spin, while the other systems show a significant spin-orbit misalignment, including at least 5 with retrograde orbits.

The solar system also provides a clue. Except for Pluto, all planets outside $1 \mathrm{AU}$ lie within $2^{\circ}$ of the ecliptic plane, while the Sun's equatorial plane is inclined by $7^{\circ}$ with respect to the ecliptic. It is not clear whether the difference between $2^{\circ}$ and $7^{\circ}$ is significant or needs an explanation.

The process of planetary system formation can be roughly divided into two stages. In the first stage, which lasts a few million years until the dissipation of the gaseous protoplanetary disc, planets are formed and undergo migration due to tidal interaction with the gaseous disc. The second stage, which lasts from when the disc has dissipated to the present, involves dynamical gravitational interactions between multiple planets if they are produced in the first stage in a sufficiently close-packed configuration (e.g., Juric \& Tremaine 2007; Chatterjee et al. 2008; Nagasawa et al. 2008), and/or secular 
interactions with a distant planet or stellar companion (Wu \& Murray 2003; Fabrycky \& Tremaine 2007). The eccentricity distribution of exoplanetary systems and the recent observational results on the spin - orbit misalignment indicate that the physical processes in the second stage play an important role in determining the properties of exoplanetary systems. Nevertheless, the importance of the first stage cannot be neglected as it sets the initial condition for the possible evolution in the second stage.

The main message of this talk is that magnetic interaction torque between a protostar and its disk can (but not always) push the stellar spin axis away from the disk axis. This implies that (i) protoplanetary disks do not have to be aligned with the stellar spin, and (ii) before few-body interaction starts (in the second stage), the planet's orbital axis may already be misaligned with the stellar spin. This talk is based on two papers, Lai, Foucart \& Lin (2011) and Foucart \& Lai (2011), in which more details and references can be found.

\section{Physical Origin of the Magnetic Warping Torque}

The interaction between a magnetic star and a disk is complex. However, the key physics related to the magnetic torques of interest here can be described robustly in a parametrized manner (see Fig. 1). The stellar magnetic field disrupts the accretion disc at the magnetospheric boundary, where the magnetic and plasma stresses balance:

$$
r_{\mathrm{in}}=\eta\left[\mu^{4} /\left(G M_{\star} \dot{M}^{2}\right)\right]^{1 / 7},
$$

where $\mu$ is magnetic moment of the protostar, $\dot{M}$ is the mass accretion rate, and $\eta$ is a dimensionless constant somewhat less than unity $(\eta \sim 0.5)$. Before being disrupted, the disc generally experiences nontrivial magnetic torques from the star. These torques are of two types: (i) A warping torque $\mathbf{N}_{w}$ which acts in a small interaction region $r_{\text {in }}<r<r_{\text {int }}$, where some of the stellar field lines are linked to the disc. These field lines are twisted by the differential rotation between the star and the disc, generating a toroidal field $\Delta B_{\phi}=\mp \zeta B_{z}^{(s)}$ from the quasi-static vertical field $B_{z}^{(s)}$ threading the disc, where $\zeta \sim 1$ and the upper/lower sign refers to the value above/below the disc plane. Since the toroidal field from the stellar dipole $B_{\phi}^{(\mu)}$ is symmetric with respect to the disc plane, the net toroidal field differs above and below the disc plane, giving rise to a vertical force on the disc. While the mean force (averaging over the azimuthal direction) is zero, the uneven distribution of the force induces a net warping torque which tends to push the orientation of the disc angular momentum $\hat{\boldsymbol{l}}$ away from the stellar spin axis $\hat{\boldsymbol{\omega}}_{s}$. The essential physics of the warping torque can also be understood from the "laboratory" toy model depicted in Fig. 1(b). (ii) A precession torque $\mathbf{N}_{p}$ which arises from the screening of the azimuthal electric current induced in the highly conducting disc. This results in a difference in the radial component of the net magnetic field above and below the disc plane and therefore in a vertical force on the disc. The resulting precession torque tends to cause $\hat{\boldsymbol{l}}$ to precess around $\hat{\boldsymbol{\omega}}_{s}$.

The two magnetic torques (per unit area) on the disc can be written as

$$
\mathbf{N}_{w}=-\left(\Sigma r^{2} \Omega\right) \cos \beta \Gamma_{w} \hat{\boldsymbol{l}} \times\left(\hat{\boldsymbol{\omega}}_{s} \times \hat{\boldsymbol{l}}\right), \quad \mathbf{N}_{p}=\left(\Sigma r^{2} \Omega\right) \cos \beta \Omega_{p} \hat{\boldsymbol{\omega}}_{s} \times \hat{\boldsymbol{l}},
$$

where $\Sigma(r)$ is the surface density, $\Omega(r)$ the rotation rate of the disc, and $\beta(r)$ is the disc tilt angle (the angle between $\hat{\boldsymbol{l}}(r)$ and the spin axis $\left.\hat{\boldsymbol{\omega}}_{s}\right)$. The warping rate and precession 

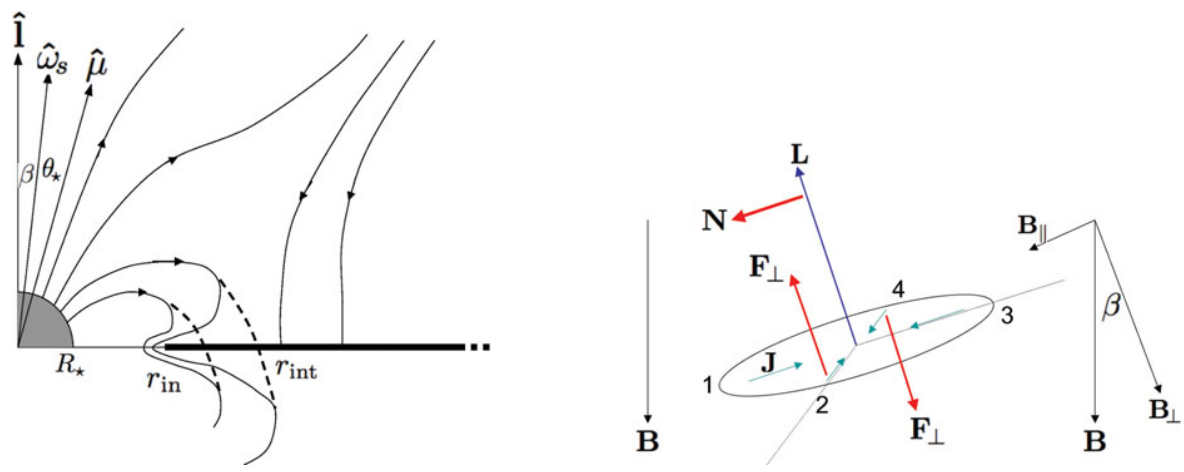

Figure 1. (a) A sketch of magnetic field configuration in a star - disc system for nonzero $\beta$ (the angle between the disc axis and the stellar spin axis) and $\theta_{\star}$ (the angle between the stellar dipole axis and the spin axis). Part of the stellar magnetic fields (dashed lines) penetrate the disc in the interaction zone between the disc inner radius $r_{\mathrm{in}}$ and $r_{\mathrm{int}}$ in a cyclic manner, while other field lines are screened out of the disc. The closed field lines are twisted by the differential rotation between the star and the disc, which leads to a magnetic braking torque and a warping torque. The screening current in the disc leads to a precessional torque. (b) A toy model for understanding the origin of the warping torque. A tilted rotating metal plate (with angular momentum $\mathbf{L}$ ) in an external magnetic field $\mathbf{B}$ experiences a vertical magnetic force around region 2 and 4 due to the interaction between the induced current $\mathbf{J}$ and the external $\mathbf{B}_{\|}$, resulting in a torque $\mathbf{N}$ which further increases the tilt angle $\beta$.

angular frequency at radius $r$ are given by

$$
\Gamma_{w}(r)=\frac{\zeta \mu^{2}}{4 \pi r^{7} \Omega(r) \Sigma(r)} \cos ^{2} \theta_{\star}, \quad \Omega_{p}(r)=-\frac{\mu^{2}}{\pi^{2} r^{7} \Omega(r) \Sigma(r) D(r)} \sin ^{2} \theta_{\star},
$$

where $\theta_{\star}$ is the angle between the magnetic dipole axis and the spin axis, and the dimensionless function $D(r)$ is somewhat less than unity.

\section{Disk Response to Magnetic Torques and Back-reaction on the Star}

In the last section, we showed that the inner region of the disc where magnetic field lines connect star and the disc and where the disc rotates faster than the star $\left(\Omega>\Omega_{s} \cos \beta\right)$ experiences a warping torque and a precessional torque. If we imagine dividing the disc into many rings, and if each ring were allowed to behave independent of each other, it would be driven toward a perpendicular state and precess around the spin axis of the central star. Obviously, real protoplanetary discs do not behave as a collection of noninteracting rings: Hydrodynamic stresses (bending waves and viscosity) provide strong couplings between different rings, and the disk will resist the inner disk warping. Detailed calculations by Foucart \& Lai (2011) showed that for most reasonable stellar/disc parameters, the steady-state disc warp is rather small because of efficient viscous damping or propagation of bending waves. Thus the inner disk direction is approximate the same as the outer disk direction.

What is happening to the stellar spin? Is there a secular change to the stellar spin direction? Note that there are a hierarchy of timescales in this problem: (1) The dynamical time associated with the spin frequency, disc rotation frequency and the beat frequency $\left|\omega_{s}-\Omega\right|$. This is much shorter than the effects of interest here. (ii) The warping/precession timescale of the inner disc, of order a few months. (iii) The disc warp evolution timescale $t_{\text {disc }}$ due to viscosity or bending wave propagation, of order 1000's of years. (iv) The stellar spin evolution timescale. The magnetic misalignment torque on the star is of the 

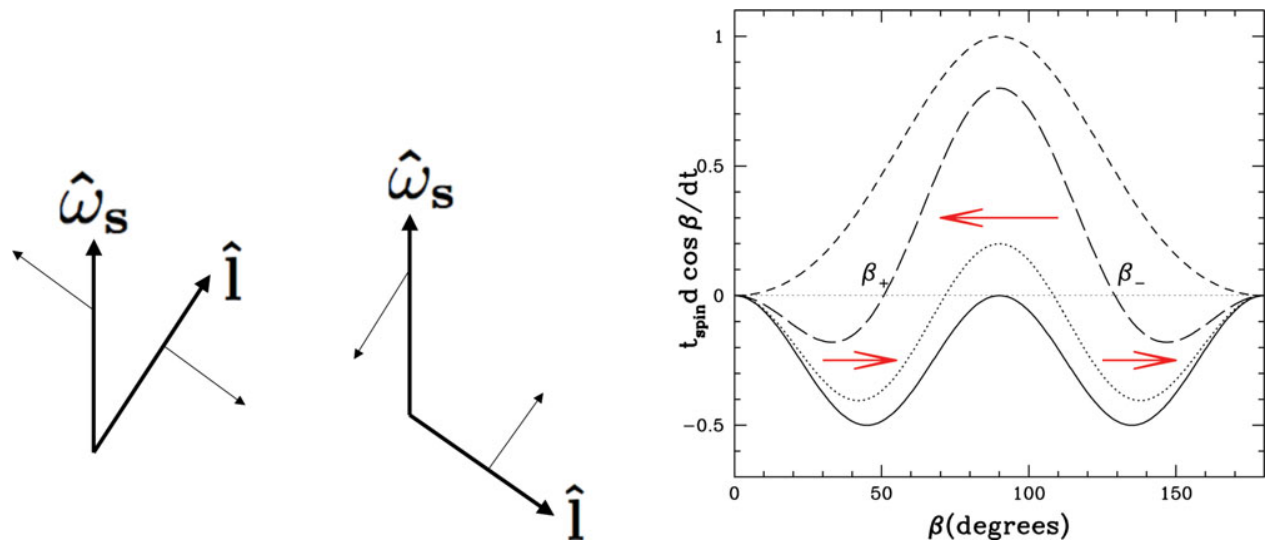

Figure 2. (a) A sketch of the effect of the magnetic warping torque. This torque tends to push the disc angular momentum axis $\hat{\boldsymbol{l}}$ toward a state perpendicular to the spin axis When $\hat{\boldsymbol{l}}$ is fixed by the outer disc, the back reaction torque tends to push the spin axis toward being misaligned with $\hat{\boldsymbol{l}}$. (b) The rate of change of the stellar inclination angle $\beta$ for a fixed disc rotation axis. From top to bottom, the four curves correspond to $(\lambda, \tilde{\zeta})=(1,0.5),(0.8,2),(0.2,2)$ and $(0,2)$, respectively. The arrows indicate the direction of $\beta$ evolution.

same order as the fiducial accretion torque $\mathcal{N}_{0}=\dot{M} \sqrt{G M_{\star} r_{\text {in }}}$. Assuming the spin angular momentum $J_{s}=0.2 M_{\star} R_{\star}^{2} \omega_{s}$, we find the spin evolution time

$$
t_{\text {spin }}=\frac{J_{s}}{\mathcal{N}_{0}}=(1.25 \mathrm{Myr})\left(\frac{M_{\star}}{1 M_{\odot}}\right)\left(\frac{\dot{M}}{10^{-8} M_{\odot} \mathrm{yr}^{-1}}\right)^{-1}\left(\frac{r_{\mathrm{in}}}{4 R_{\star}}\right)^{-2} \frac{\omega_{s}}{\Omega\left(r_{\mathrm{in}}\right)} .
$$

We are interested in the evolution of the stellar spin direction on timescale of order $t_{\text {spin }}$.

We can now consider the back-reaction torque from the disk on the stellar spin. For simplicity, let us assume small warps so that the disk is flat (averaged over the dynamical timescale). If the magnetic torque were the only torque acting on the star, then it is clear (see Fig. 2) that even if initially the stellar spin axis is approximately (but not perfectly) aligned with the disc axis, given enough time, the stellar spin axis will evolve towards the perpendicular state and even the retrograde state.

However, there are other torques acting on the star which counter-act the warping torque. In general, the evolution equation for the spin angular momentum of the star, $J_{s} \hat{\boldsymbol{\omega}}_{s}$, can be written in the form

$$
\frac{d}{d t}\left(J_{s} \hat{\boldsymbol{\omega}}_{s}\right)=\mathcal{N}=\mathcal{N}_{l}+\mathcal{N}_{s}+\mathcal{N}_{w}+\mathcal{N}_{p}
$$

Here $\mathcal{N}_{l}$ represents the torque component (including the accretion torque) that is aligned with the inner disc axis:

$$
\mathcal{N}_{l}=\lambda \dot{M}\left(G M_{\star} r_{\text {in }}\right)^{1 / 2} \hat{\boldsymbol{l}},
$$

where $\lambda \lesssim 1$ is a dimensionless parameter. The term $\mathcal{N}_{s}=-\left|\mathcal{N}_{s}\right| \hat{\boldsymbol{\omega}}_{s}$ represents a spindown torque carried by a wind/jet from the open field line region of the star. The term $\mathcal{N}_{w}$ and $\mathcal{N}_{p}$ represent the back-reactions of the warping and precessional torques, respectively. Note that both $\mathcal{N}_{w}$ and $\mathcal{N}_{p}$ are of order $\mu^{2} / r_{\text {in }}^{3}$, which does not directly depend on $\dot{M}$. But since $r_{\text {in }}$ depends on $\dot{M}$, we find that both $\mathcal{N}_{w}$ and $\mathcal{N}_{p}$ are of the same order of magnitude as the fiducial accretion torque $\mathcal{N}_{0}$. The inclination angle of the 
stellar spin relative to the disc evolves according to the equation

$$
\frac{d}{d t} \cos \beta=\frac{\mathcal{N}_{0}}{J_{s}} \sin ^{2} \beta\left(\lambda-\tilde{\zeta} \cos ^{2} \beta\right), \quad \text { with } \tilde{\zeta}=\frac{\zeta^{\prime} \cos ^{2} \theta_{\star}}{6 \eta^{7 / 2}} .
$$

Equation (3.4) is our key result. It reveals the following behavior for the evolution of $\beta$ (see Fig. 2): (i) For $\lambda=0$, equation (3.4) describes the effect of the magnetic warping torque acting alone on the star. This torque always pushes the stellar spin toward antialignment with $\hat{\boldsymbol{l}}$. (ii) For $\tilde{\zeta} / \lambda<1$ : Regardless of the initial $\beta$, the spin always evolves towards alignment. (iii) For $\tilde{\zeta} / \lambda>1$ : There are two possible directions of $\beta$ evolution, depending on the initial value of $\beta$. The condition $d \cos \beta / d t=0$ yields two "equilibrium"

states $\left(\beta_{+}\right.$and $\left.\beta_{-}\right)$, given by $\cos \beta_{ \pm}= \pm \sqrt{\lambda / \tilde{\zeta}}$. Of the two equilibria, one is stable $\left(\beta_{+}\right)$ and the other is unstable. For $\beta(t=0)<\beta_{-}$, the system will evolve towards a misaligned prograde state $\beta_{+}$; for $\beta(t=0)>\beta_{-}$, the system will evolve towards the anti-aligned state $\left(\beta=180^{\circ}\right)$.

\section{Discussion}

Note that there is a 90-degree barrier: Starting from a small angle $\beta$, the systen cannot evolve into retrograde if the outer disk orientation is fixed. However, it is possible to produce retrograde systems in two ways: (i) If the outer disk changes direction (e.g., due to external perturbers/flybys); (ii) If the initial condition is retrograde: this may be the case if we consider disk formation in turbulent star-forming clouds, as suggested by Bate et al. (2010). But note that even in this scenario, the magnetic misalignment effect discussed above is important - without it, the stellar spin would align with the disk axis on a short timescale. In fact, starting from a random distribution of the initial relative orientation between the spin and disk axes, we can calculate the evolution of the stellar obliquity distribution as a function of time. For "weak" magnetic torques $(\tilde{\zeta} / \lambda<1)$, a distribution peaked at $\beta=0$ is produced. For "strong" magnetic torques $(\tilde{\zeta} / \lambda>1)$, we produce a bi-modal distribiton: one peak is at $\beta_{+}$, another at $\beta=180^{\circ}$ (see Lai et al. 2010).

How to test the effects discussed above? One way is to measure the spin-orbit angles for systems with two transiting planets (Kepler-9 is an example). Another is to measure the orientation of the stellar spin and its disk. Recently, Watson et al. (2011) carried out such an analysis for several debris disc systems and found no significant difference between $\sin i_{\star}$ and $\sin i_{\text {disc }}$. Note that $i_{\star}=i_{\text {disc }}$ does not necessarily imply alignment between the spin axis and the disc axis. Also, systematic uncertainties in estimating $i_{\text {disc }}$ need to be taken into account. For example, for HD 22049 (one of the best cases studied by Watson et al.), the disc inclination is consistent with face-on $\left(i_{\text {disc }} \lesssim 25^{\circ}\right.$; Backman et al. 2009). It would be useful to do this for classical T Tauri stars.

\section{References}

Backman, D., et al. 2009, ApJ, 690, 1522

Chatterjee, S., et al. 2008, ApJ, 686, 580

Fabrycky, C. \& Tremaine, S. 2007, ApJ, 669,1298

Foucart, F. \& Lai, D. 2011, MNRAS, 412, 2799

Juric, M. \& Tremaine, S. 2008, ApJ, 686, 603

Lai, D., Foucart, F., \& Lin, D. N. C.. 2011, MNRAS, 412, 2790

Nagasawa, M., Ida, S., \& Bessho, T. 2008, ApJ, 678, 498

Wu, Y. \& Murray, N. W. 2003, ApJ, 589, 605

Watson, C. A., et al. 2011, MNRAS, 413, L71 